\title{
EQUILIBRIUM AND NON-EQUILIBRIUM ISING MODELS BY MEANS OF PCA
}

\author{
Carlo Lancia ${ }^{1,2}$ and Benedetto Scoppola ${ }^{1}$ \\ ${ }^{1}$ Università di Roma 'Tor Vergata' \\ ${ }^{2} \mathrm{TU} / \mathrm{e}$, Technische Universiteit Eindhoven
}

January 12, 2021

\begin{abstract}
We propose a unified approach to reversible and irreversible PCA dynamics, and we show that in the case of 1D and 2D nearest neighbour Ising systems with periodic boundary conditions we are able to compute the stationary measure of the dynamics also when the latter is irreversible We also show how, according to [DPSS 12], the stationary measure is very close to the Gibbs for a suitable choice of the parameters of the PCA dynamics, both in the reversible and in the irreversible cases. We discuss some numerical aspects regarding this topic, including a possible parallel implementation.
\end{abstract}

\section{INTRODUCTION}

In this paper we propose a connection between two different subjects that have been quite studied over the last two decades, that is, the general study of non-equilibrium statistical mechanics and the description of equilibrium statistical mechanics with the specific use of a Probabilistic Cellular Automaton (PCA). Starting from a series of seminal papers, e.g. [GKLM89. LMS90, MS93], a certain effort has been spent in order to give a dynamical description of the equilibrium statistical mechanics by means of PCAS. A PCA is a discrete-time Markov chain (MC) on a product space $S^{V}$ such that the transition probability $P(\sigma, \tau)$ is a product measure

$$
P(\sigma, \tau)=\prod_{i \in V} p_{i}\left(\tau_{i} \mid \sigma\right)
$$

where $p_{i}\left(\tau_{i} \mid \sigma\right)$ is a probability on $S$ for all $i \in V$ and $\sigma \in S^{V}$. Given a probability measure $\mu$ on $S^{V}$, the question is whether a PCA may be constructed whose stationary measure is $\mu$. In the context of the equilibrium statistical mechanics we are obviously interested to the case in which $\mu$ is a Gibbs measure. It is well known that such a problem is rather difficult. For instance, in [KV80] it is shown that no PCA can be designed in such a way to be reversible with respect to the stationary distribution of a $2 \mathrm{D}$ Ising model. In [LMS90] and [CNo3] a PCA is introduced whose invariant, reversible measure $\pi$ is related to the Ising model as follows. The projection of $\pi$ to the even sites, i.e., those $(i, j) \in \mathbb{Z}^{2}$ with $i+j$ even, coincides with the same projection of the Ising model, and the same holds for odd sites. However, opposite to the Ising model, spins at even sites are independent under the measure $\pi$ of those at odd sites. Recently, one of the authors has proved in [DPSS12] that the PCA from [CNo3] can be simply modified in order to provide a way to approximately sample from a Gibbs measure. The sense of this statement will be clarified in Section 2. This result was 
achieved following some ideas first introduced in [ISSo7] and later exploited in [SCO11] to provide an easy example of cutoff.

On the other hand, one of the main subjects of the recent research in statistical mechanics has been the study of non-equilibrium statistical mechanics, in particular when a stationary state can be defined. Several different approaches have been proposed, see e.g. [GC95a, GC95b], [JL10] and the references therein. In contrast with the case of equilibrium statistical mechanics, where a well defined recipe, the Gibbs measure, is always available, in the non-equilibrium case the subject is far from a complete and general understanding. The study of the stationary measure in the non-equilibrium context can not leave aside the dynamics of the process whereas the Gibbs recipe has exactly this advantage.

In this paper we propose, starting from the results in [DPSS12], a unified description of equilibrium and non-equilibrium statistical mechanics in terms of PCA. It turns out that such a description gives to the chance to compute explicitly the stationary state of a set of the statistical mechanics models, including the nearest neighbour Ising model in 1 and 2 dimensions. An extension of the class of system for which this approach give rise to explicit computations seems very likely with the use of relatively easy arguments. The paper is organised as follows. In Section 2 we will define, according to [DPSS12], a class of reversible and irreversible PCA dynamics. We will prove that if a weak balance condition is verified then we are able to explicitly write the unique stationary measure of the chain. In Section 3 we show that the Ising systems introduced in Section 2 are weakly balanced whenever periodic boundary conditions are imposed. Finally, in Section 4 we present some numerical/simulative aspects of PCAs. In particular, we show that in the irreversible case a stationary current is present in the system, and under suitable conditions what we call Ising waves arise.

2 REVERSIBLE AND IRREVERSIBLE PCA DYNAMICS

Let us consider a two-body spin system, defined by an Hamiltonian of the form

$$
H(\sigma)=-\sum_{(x, y)} J_{\{x, y\}} \sigma_{x} \sigma_{y},
$$

where $\Lambda$ is a finite set, $\sigma \in \mathcal{X}=\{-1,+1\}^{\Lambda}$, and the sum in (2.1) is extended to the unordered pairs $\{x, y\}$ with $x, y \in \Lambda$. The Gibbs measure associated to the Hamiltonian (2.1) is then

$$
\pi_{G}(\sigma)=\frac{e^{-H(\sigma)}}{Z}
$$

where $Z=\sum_{\sigma} e^{-H(\sigma)}$. It is possible to construct Markovian, ergodic dynamics having stationary measure is precisely $\pi_{G}(\sigma)$. The long run behaviour of the chain can then be used to sample from $\pi_{G}(\sigma)$. Those Markovian dynamics are typically based on single spin-flip transition probabilities, see for instance [Bre99, LPWo9] and the references therein.

One of the authors has recently proved in [DPSS12] that an approximate sampling of Gibbs measure 2.2. can be actually achieved also by means of a reversible PCA dynamics. Consider the following Hamiltonian, defined on pairs of configurations $(\sigma, \tau)$,

$$
H(\sigma, \tau)=-\sum_{(x, y)} J_{x, y} \sigma_{x} \tau_{y}-q \sum_{x} \sigma_{x} \tau_{x}
$$

where

$$
J_{x, y}=J_{y, x}=\frac{J_{\{x, y\}}}{2}, \quad \forall x, y \in \Lambda .
$$

The last term in the Hamiltonian 2.3, proportional to the parameter $q>0$, represents an inertial term, i.e., the tendency of the system to remain in 
the current state $\sigma$. We next define a homogeneous MC whose transition probabilities are

$$
P(\sigma, \tau)=\frac{e^{-H(\sigma, \tau)}}{Z_{\sigma}}
$$

where

$$
Z_{\sigma}=\sum_{\tau} e^{-H(\sigma, \tau)} .
$$

From (2.4) $H\left(\sigma, \sigma^{\prime}\right)=H\left(\sigma^{\prime}, \sigma\right)$, thus the Hamiltonian (2.3) is symmetric for the exchange $\sigma \leftrightarrow \sigma^{\prime}$. It is then immediate to see that such a MC is ergodic and reversible with respect to the measure

$$
\pi(\sigma)=\frac{Z_{\sigma}}{Z}
$$

where $Z=\sum_{\sigma} Z_{\sigma}$. The following result holds

$$
\begin{gathered}
\text { THEOREM } 1 \text { [DPSS12] If } e^{-2 q}=o\left(|\Lambda|^{\frac{1}{2}}\right) \text { as }|\Lambda| \rightarrow \infty \text { then } \\
\qquad \lim _{\Lambda \rightarrow \infty} d_{T V}\left(\pi, \pi_{G}\right)=0
\end{gathered}
$$

where $d_{T V}(\cdot, \cdot)$ is the usual total-variation distance and $\pi_{G}$ is given by 2.1- 2.2 .

Remark 1. The long run behaviour of the collective dynamics defined by 2.5 can be used to perform an approximate sampling of a Gibbs distribution in the sense of 2.8). Note that in [DPSS12] the limit 2.8) is obtained without making any use of reversibility.

Having in mind a generalisation of this picture to irreversible MCs, let us define a non-symmetric Hamiltonian on pairs of configurations by simply taking [2.3], where now $J_{x, y} \neq J_{y, x}$.

Definition 1. Whenever $J_{x, y} \neq 0 \Rightarrow J_{y, x}=0$ we will say that the spin system 2.3 is completely asymmetric.

The new chain will evolve according to 2.5 . Due to the lack of symmetry of the interaction $J_{x, y}$, the MC is no longer reversible with respect to 2.7 because $H(\sigma, \tau) \neq H(\tau, \sigma)$ and the detailed balance condition is not satisfied. However, we can still consider $\pi(\sigma)$ given by [2.7) and compute $\sum_{\sigma} \pi(\sigma) P(\sigma, \tau)$. This yields

$$
\sum_{\sigma} \pi(\sigma) P(\sigma, \tau)=\sum_{\sigma} \frac{Z_{\sigma}}{Z} \frac{e^{-H(\sigma, \tau)}}{Z_{\sigma}}=\sum_{\sigma} \frac{e^{-H(\sigma, \tau)}}{Z} .
$$

PROPOSITION 2 Consider the following MC

$$
\begin{aligned}
P(\sigma, \tau) & =\frac{e^{-H(\sigma, \tau)}}{Z_{\sigma}}, \\
H(\sigma, \tau) & =-\sum_{(x, y)} J_{x, y} \sigma_{x} \tau_{y}-q \sum_{x} \sigma_{x} \tau_{x}, \\
Z_{\sigma} & =\sum_{\tau} e^{-H(\sigma, \tau)},
\end{aligned}
$$

where no assumption is made on $J_{x, y}$. Suppose the following Weak Balance Condition (wBC) holds

$$
\sum_{\tau} e^{-H(\sigma, \tau)}=\sum_{\tau} e^{-H(\tau, \sigma)}, \quad \forall \sigma \in \mathcal{X} .
$$

Then the probability distribution

$$
\pi(\sigma)=\frac{Z_{\sigma}}{Z} \quad \text { with } \quad Z=\sum_{\sigma} Z_{\sigma},
$$

is the stationary distribution of the chain. 
Proof. By 2.9] and 2.13),

$$
\sum_{\sigma} \pi(\sigma) P(\sigma, \tau)=\sum_{\tau} \frac{e^{-H(\tau, \sigma)}}{Z}=\frac{Z_{\tau}}{Z}=\pi(\tau) .
$$

Therefore (2.14) is stationary.

Remark 2. The швс 2.13 determines a class of MCs for which the stationary distribution is (2.14), some examples within this class are presented in Section 3 The fulfilment of the $\mathrm{WBC}$ is indeed a weaker requirement than the detailed balance principle, which holds for the reversible dynamics due to 2.4 .

We end the present section with a couple of important remarks

Remark 3. Given a function $g: \mathcal{X} \times \mathcal{X} \rightarrow \mathbb{R}$, its expectation with respect to the stationary measure over pairs of subsequent configurations is

$$
\langle g(\sigma, \tau)\rangle=\sum_{\sigma, \tau} \pi(\sigma) P(\sigma, \tau) g(\sigma, \tau)=\sum_{\sigma, \tau} \frac{e^{-H(\sigma, \tau)} g(\sigma, \tau)}{Z} .
$$

Hence, the two-step stationary measure of the dynamics is Gibbsian in the sense of 2.16.

Remark 4. Given a function $g: \mathcal{X} \times \mathcal{X} \rightarrow \mathbb{R}$, consider

$$
\mathcal{J}(g)=\langle g(\sigma, \tau)-g(\tau, \sigma)\rangle .
$$

Whenever $\mathcal{J}(g) \neq 0$ we see a current of the function $g$. In the reversible case $\mathcal{J}(g)$ is identically zero for each $g$, while in the irreversible case it may be in general different from zero.

\section{WEAKLY BALANCED ISING IRREVERSIBLE SYSTEMS}

We prove the WBC 2.13) for some examples of $1 \mathrm{D}$ and 2D nearest neighbour Ising systems. Similar proofs can be done in more general cases.

\subsection{Weak balance for $1 D$ nearest neighbour Ising systems}

The Hamiltonian of the 1D nearest neighbour Ising model is given by

$$
H(\sigma)=-\sum_{i=1}^{L} J_{i} \sigma_{i} \sigma_{i+1} .
$$

Here we assume that the strength of the interaction $J_{i}$ may in general depend on the site $i$. The sum on $i$ may be performed using either periodic boundary conditions or empty boundary condition. In the first case sites 1 and $L+1$ coincide (Ising system on the circle). In the second case $J_{L}=0$ (Ising system on the segment).

We can introduce a PCA reversible dynamics for this model starting from the pair Hamiltonian

$$
H(\sigma, \tau)=-\sum_{i=1}^{L}\left[\frac{J_{i}}{2}\left(\sigma_{i} \tau_{i+1}+\tau_{i} \sigma_{i+1}\right)+q \sigma_{i} \tau_{i}\right],
$$

and then defining the PCA dynamics with the transition probabilities

$$
P(\sigma, \tau)=\frac{e^{-H(\sigma, \tau)}}{Z_{\sigma}} .
$$

Both periodic and empty boundary conditions can be considered for the sum (3.2). This reversible PCA dynamics can be generalised to an irreversible 
case in two ways. The first possibility is to define a completely asymmetric system by the pair Hamiltonian

$$
H(\sigma, \tau)=-\sum_{i=1}^{L}\left[J_{i} \sigma_{i} \tau_{i+1}+q \sigma_{i} \tau_{i}\right] .
$$

This is a particularly simple irreversible system, and the WBC can be directly checked in one line

$$
\begin{aligned}
\sum_{\tau} e^{-H(\sigma, \tau)} & =2^{L} \prod_{i} \cosh \left(J_{i-1} \sigma_{i-1}+q \sigma_{i}\right) \\
& =2^{L} \prod_{i} \cosh \left(J_{i-1} \sigma_{i}+q \sigma_{i-1}\right)=\sum_{\tau} e^{-H(\tau, \sigma)},
\end{aligned}
$$

where in the second equality we have used the parity of the hyperbolic cosine. We will now compute $Z$ using the following elementary identities

$$
\begin{aligned}
& \cosh (a+b)=\cosh a \cosh b+\sinh a \sinh b, \\
& \sinh (a+b)=\sinh a \cosh b+\cosh a \sinh b .
\end{aligned}
$$

When the system is defined on the circle,

$$
Z=\sum_{\sigma, \tau} e^{-H(\sigma, \tau)}=(\cosh q)^{L} \prod_{i} \cosh J_{i}+(\sinh q)^{L} \prod_{i} \sinh J_{i},
$$

by (3.6) and (3.7), and by the parity of the hyperbolic sine and cosine. When the system is defined on the segment

$$
Z=\sum_{\sigma, \tau} e^{-H(\sigma, \tau)}=(\cosh q)^{L} \prod_{i} \cosh J_{i} .
$$

In this simple 1D case it is also easy to exhibit a function $g$ such that $\mathcal{J}(g) \neq 0$. Let us take for instance the family $g_{i}(\sigma, \tau)=\sigma_{i} \tau_{i+1}$. For the Ising system on the segment,

$$
\begin{aligned}
\mathcal{J}\left(g_{i}\right)= & \left\langle\sigma_{i} \tau_{i+1}-\tau_{i} \sigma_{i+1}\right\rangle=Z^{-1} \sum_{\sigma, \tau} e^{-H(\sigma, \tau)}\left(\sigma_{i} \tau_{i+1}-\tau_{i} \sigma_{i+1}\right) \\
= & Z^{-1} 2^{L} \sum_{\sigma}\left[\sigma_{i} \sinh \left(J_{i} \sigma_{i}+q \sigma_{i+1}\right) \prod_{l \neq i} \cosh \left(J_{l} \sigma_{l}+q \sigma_{l+1}\right)\right. \\
& \left.-\sigma_{i+1} \sinh \left(J_{i-1} \sigma_{i-1}+q \sigma_{i}\right) \prod_{l \neq i} \cosh \left(J_{l-1} \sigma_{l-1}+q \sigma_{l}\right)\right] \\
= & \tanh J_{i}\left(1-\tanh ^{2} q\right) .
\end{aligned}
$$

The second option to generalise the PCA dynamics defined by the Hamiltonian (3.2) is to define a partially asymmetric system. The proof of the швс (2.13) relies on the hypothesis that the system is translationally invariant. As such, the system has to be defined on the circle with $J_{i}=J$ for all $1 \leq i \leq L$. The pair Hamiltonian then becomes

$$
H(\sigma, \tau)=-\sum_{i=1}^{L}\left[\alpha J \sigma_{i} \tau_{i+1}+(1-\alpha) J \tau_{i} \sigma_{i+1}+q \sigma_{i} \tau_{i}\right],
$$

where $0<\alpha<1$. The proof of $\mathrm{WBC}$ for the $2 \mathrm{D}$ version of $(3.4)$ is presented in Section 3.2 . The actual proof of the WBC for (3.11) is similar with respect to that - but much easier. It is left as an exercise to the reader.

\subsection{Weak balance for $2 D$ nearest neighbour completely asymmetric Ising systems}

We now consider the translationally invariant Ising model on a 2D square lattice $\Lambda$ with $N \times N$ sites, with periodic boundary conditions. To this purpose we introduce some notation. The configuration $\sigma \in\{-1,+1\}^{\Lambda}$ has 
value $\sigma_{i, j}$ in the site $x=(i, j)$ of the torus $\Lambda$. The Hamiltonian of the system is defined by the expression

$$
H(\sigma)=-\sum_{\substack{\{(i, j),(k, l)\} \\ \text { n.n. pairs }}} J \sigma_{i, j} \sigma_{k, l},
$$

that we can rewrite more explicitly as

$$
H(\sigma)=-J \sum_{i, j=1}^{N}\left(\sigma_{i, j} \sigma_{i, j+1}+\sigma_{i j} \sigma_{i+1, j}\right) .
$$

Similarly to the previous section, the reversible PCA dynamics associated to 3.13 is determined by the pair Hamiltonian

$$
\begin{aligned}
H(\sigma, \tau)= & -\frac{J}{2} \sum_{i, j=1}^{N}\left(\sigma_{i, j} \tau_{i, j+1}+\sigma_{i, j+1} \tau_{i, j}+\sigma_{i+1, j} \tau_{i, j}+\sigma_{i j} \tau_{i+1, j}\right) \\
& -q \sum_{i, j=1}^{N} \sigma_{i, j} \tau_{i, j} .
\end{aligned}
$$

According to (2.5) and (2.6), the Hamiltonian above defines an homogeneous MC, which is again ergodic and reversible with respect to the measure $\pi(\sigma)$, given by formulas (2.7) and (3.14).

To generalise this picture to irreversible MCs, we define an irreversible PCA considering a completely asymmetric version of the dynamics above. We define the following Hamiltonian

$$
H(\sigma, \tau)=-J \sum_{i, j=1}^{N}\left(\sigma_{i, j} \tau_{i, j+1}+\sigma_{i, j} \tau_{i+1, j}\right)-q \sum_{i, j=1}^{N} \sigma_{i, j} \tau_{i, j}
$$

Proposition 3 The irreversible PCA defined as in $(2.5)-(2.6)$ by the Hamiltonian 3.15 is weakly balanced, i.e.,

$$
\sum_{\tau} e^{-H(\sigma, \tau)}=\sum_{\tau} e^{-H(\tau, \sigma)}, \quad \forall \sigma \in \mathcal{X} .
$$

Hence, $\pi(\sigma)=\frac{Z_{\sigma}}{Z}$ is the stationary measure of such an irreversible PCA.

Proof. By direct computation we have that

$$
\begin{aligned}
& \sum_{\tau} e^{-H(\sigma, \tau)}=: Z_{\sigma}^{\rightarrow}=2^{|\Lambda|} \prod_{i, j} \cosh \left(J\left(\sigma_{i-1, j}+\sigma_{i, j-1}\right)+q \sigma_{i, j}\right), \\
& \sum_{\tau} e^{-H(\tau, \sigma)}=: Z_{\sigma}^{\leftarrow}=2^{|\Lambda|} \prod_{i, j} \cosh \left(J\left(\sigma_{i+1, j}+\sigma_{i, j+1}\right)+q \sigma_{i, j}\right) .
\end{aligned}
$$

The parity of the hyperbolic cosine then yields

$$
\cosh \left(J\left(\sigma_{i \pm 1, j}+\sigma_{i, j \pm 1}\right)+q \sigma_{i, j}\right)= \begin{cases}\cosh (2 J+q) & \text { if } \sigma_{i \pm 1, j}=\sigma_{i, j \pm 1}=\sigma_{i, j}, \\ \cosh (2 J-q) & \text { if } \sigma_{i \pm 1, j}=\sigma_{i, j \pm 1}=-\sigma_{i, j} \\ \cosh (q) & \text { if } \sigma_{i \pm 1, j} \neq \sigma_{i, j \pm 1} .\end{cases}
$$

The three different values depend on the configuration $\sigma$ when it is represented in terms of Peierls contours. Indeed, once a configuration $\sigma$ and the corresponding set of Peierls contour $\Gamma$ are chosen, then the contribution of the single term $i, j$ appearing in the products of 3.17) (resp. 3.18) depends on the contribution the dual edges below and to the left (resp. above and to the right) of the site $i, j$. If none of them belongs to any Peierls contour, the contribution will be $\cosh (2 J+q)$; if both of them belong to some Peierls contour, then the contribution will be $\cosh (2 J-q)$; if exactly one of them belongs to a Peierls contour and the other does not, then the contribution will be $\cosh (q)$. Hence, the first part of the Proposition follows simply noting that for all configurations of Peierls contours the number of elbows in the SW direction is the same of the number of elbows NE-oriented. 
Remark 5. Remark 1 implies that Theorem 1 holds also in this case. Thus, if $e^{-2 q}=o\left(|\Lambda|^{\frac{1}{2}}\right)$ as $|\Lambda| \rightarrow \infty$ then $\pi(\sigma)$, defined by 2.7 and 3.15, tends asymptotically in total-variation distance to the Gibbs measure $\pi_{G}(\sigma)$, defined by (2.2) and (3.13).

4 PARAllelization, PHASE TRANSition, ISING WAVES

In this section we present some numerical studies about the 2D Ising model PCA we have discussed in Section 3.2 above, that is, the discrete-time MC defined by (2.5)-2.6. Both (3.14) and (3.15) will be considered as the Hamiltonian of the system.

MCS can be easily implemented and simulated on any computer using the socalled random mapping representation, see e.g. [Hägo2]. Random processes of the kind of PCA can be simulated using the very same approach. The product form of the transition matrix (1.1) ensures that the spins flip independently, so a unique random map will serve all the spins. To fix the ideas, let us consider the reversible PCA defined by (2.5), 2.6) and (3.14). If we define the local field to be

$$
h_{i, j}(\sigma)=\frac{J}{2}\left(\sigma_{i-1, j}+\sigma_{i+1, j}+\sigma_{i, j-1}+\sigma_{i, j+1}\right) .
$$

Then equation (3.14) becomes

$$
H(\sigma, \tau)=-\sum_{i, j=1}^{N}\left[h_{i, j}(\sigma)+q \sigma_{i, j}\right] \tau_{i, j} .
$$

Thus, the transition probabilities 2.5- 2.6 become

$$
P(\sigma, \tau)=\prod_{i, j} \frac{\exp \left[\tau_{i, j}\left(h_{i, j}(\sigma)+q \sigma_{i, j}\right)\right]}{2 \cosh \left(h_{i, j}(\sigma)+q \sigma_{i, j}\right)} .
$$

The update procedure of a spin at vertex $(i, j)$ is sketched in the pseudocode given by Listing 1 The number of floating point operations required for the update of a single spin is very low. Indeed, the problem of simulating the evolution of such a MC is not particularly complex, involving only simple operations on many data elements. This is particularly suited for a Graphics Processing Unit (GPU) because it closely resembles the operations involved in graphic applications. Moreover, from (1.1) there is no dependency in the probabilistic update rule for the evolution of the spins. Therefore they can be updated simultaneously, making the problem embarrassingly parallel. The natural choice to efficiently implement such a model seems to be Compute Unified Device Architecture (CUDA).

CUDA maps geometry onto blocks of threads executing simultaneously. It is then natural to map a configuration $\sigma$, stored as a square matrix of size $N \times N$,

Listing 1: Update procedure for the spin in site $(i, j)$

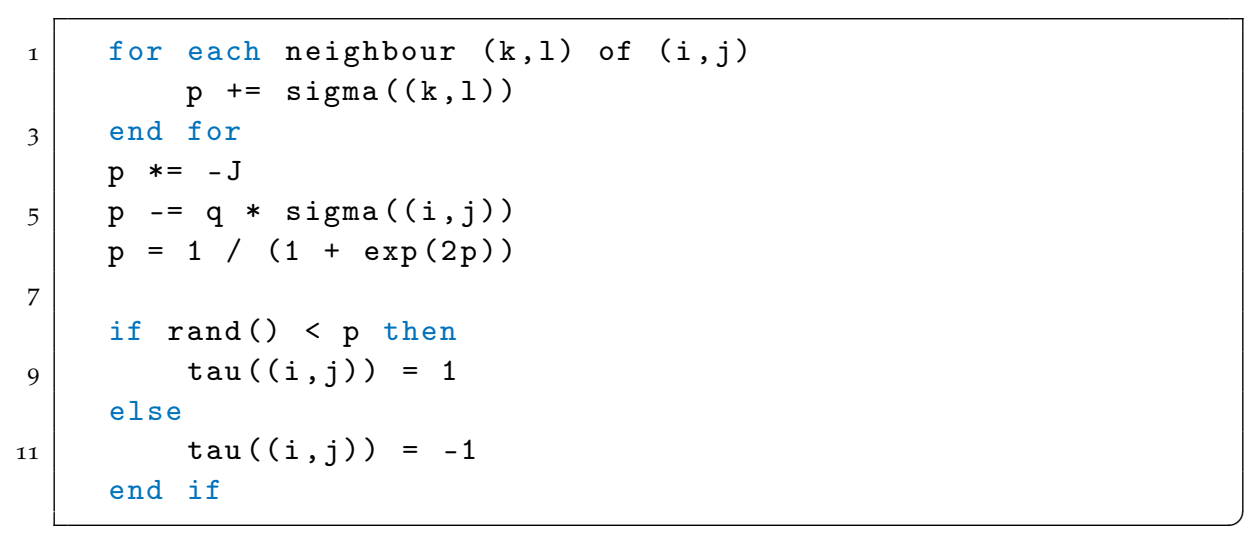


onto a square grid of blocks. Each block is able to run multiple threads, simultaneously updating a portion of the configuration matrix $\sigma$. The update rule (1.1) ensures that no inter-thread dependencies arise as the transition probabilities depend only on the previous time-step configuration. The only aspect that must be carefully regarded is the memory access, because the memory is the main potential performance inhibitor in GPUs. It is hence very important to optimise for memory access. Dividing the two dimensional matrix into a set of square tiles is a good strategy to efficiently use the GPU's shared memory and minimise the number of global memory reads. However, the presence of periodic boundary conditions causes a non uniform access pattern on the boundary, that is, the impossibility to achieve coalesced memory and so high memory bandwidth. This latter issue disappears if one considers non-periodic boundary conditions.

Figure 1 shows the execution time of 500 successive transitions of the PCA for different values of the number of sites $N$ in each dimension. In particular, we see that for a square lattice of size $14000 \times 14000$ the execution time is less than one minute. Together with Theorem 1 this paves the way for an extremely sped-up Gibbs sampling.

Let us now consider the irreversible PCA defined by $(2.5),(2.6)$ and $(3.15)$, with initial state $\sigma_{i, j}=-1$ for all sites $(i, j)$. To have fairly readable pictures, we set the size of the square lattice equal to $50 \times 50$. The evolution of the dynamics for $J=0.5$ and $q=1.0$ is presented in Figure 非, whereas the evolution for $J=0.5$ and $q=3.0$ Figure $2 \mid$. In the latter case the product $|\Lambda|^{\frac{1}{2}} e^{-2 q}$ is small, then from Theorem 1 we may expect the typical configurations of PCAS and serial Glauber dynamics to be rather alike. Indeed, Figure $2 p$ displays roundish clusters of +1 spins, quite similar to the standard Ising droplets. Clearly the total asymmetry of the interaction is still visible as the droplets present a moderate degree of stretching along the SE direction. The droplets stretch is even more evident in Figure 非, where the contributions to the Hamiltonian due to the local field and to the inertial term are comparable. In Figure 3 the value of $q$ is fixed to the value $\frac{1}{2}$. For a large value of $J$ the system exhibits spontaneous magnetization (Fig. 3 b), whereas for a small value of $q$ the system exhibit a paramagnetic behaviour (Fig. 非. Figure 3 then suggests the presence of a phase transition, and a comparison of Figure 非 with Figures 国 $\mathrm{b}$ clearly implies that the critical temperature, if any exists, must be function of both $J$ and $q$.

The last argument has shown the need to explore the whole plane $J, q$. A region of this plane we find rather interesting is that for $J$ large and $q$ small. Here the spin-flipping is not hampered by the contribution $-q \sigma_{i, j} \tau_{i, j}$, so the alignment of a spin with its souhtern and western nearest neighbour costs virtually nothing. Figure 4 shows the evolution for $J=1.5$ and $q=0.1$. Due to the mechanism we have just described, the droplets steadily drift in the NE direction. The constant drift suggests the presence of a current of a family of functions

$$
g_{i, j}(\sigma, \tau)=\tau_{i, j}\left(\sigma_{i-1, j}+\sigma_{i, j-1}\right),
$$

which generalise to the 2D case what we have already discovered in 3.10. Figure 4 also suggests that there is another mechanism at work along the mentioned drift. Due to the total asymmetry of the interaction, the growth of the droplets along the SW direction is preferred. We propose for such elongated, drifting droplets the name of Ising waves. 


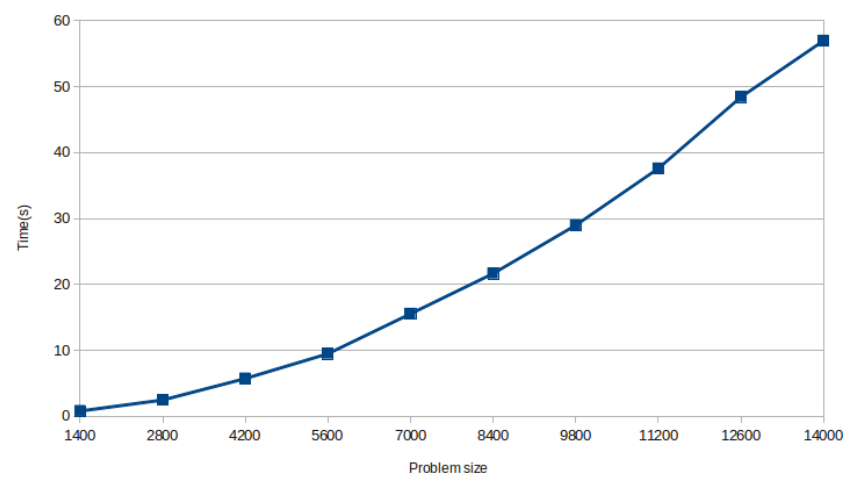

Figure 1: Execution time of 500 steps of the PCA dynamics defined by (2.5), 2.6 and (3.14). Different values of $N$ are considered. Simulations were run on a Nvidia Tesla K20s GPU.
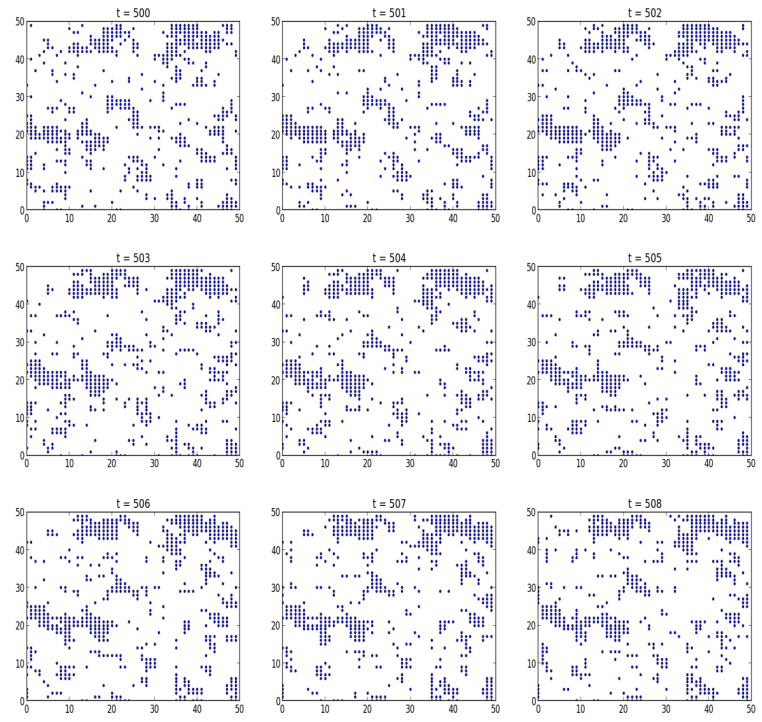

(a) $q=1.0$
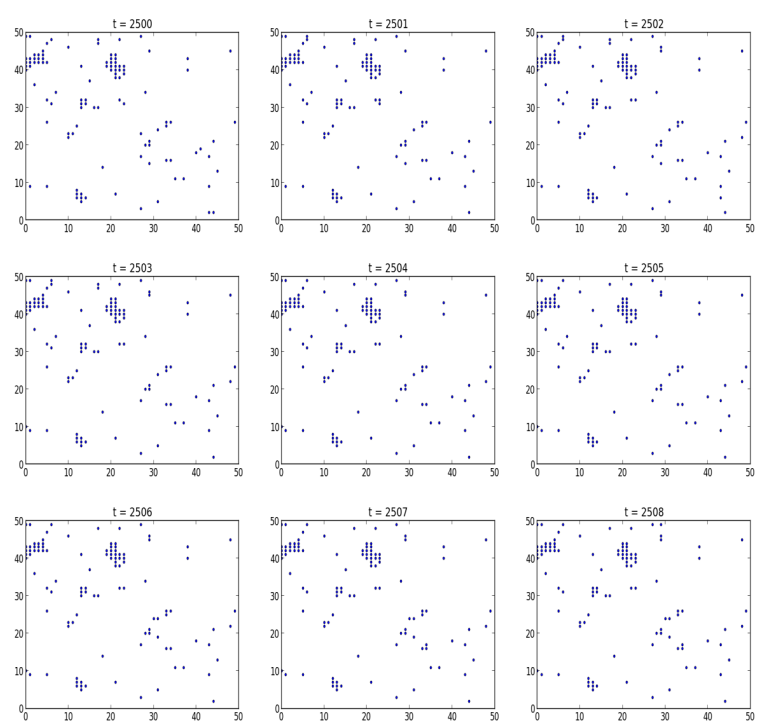

(b) $q=3.0$

Figure 2: Simulation of nine successive steps of the PCA dynamics defined by 2.5 , 2.6) and 3.15 , with $N=50, J=0.5$ and different values of $q$. Blue dots represents +1 spins. 

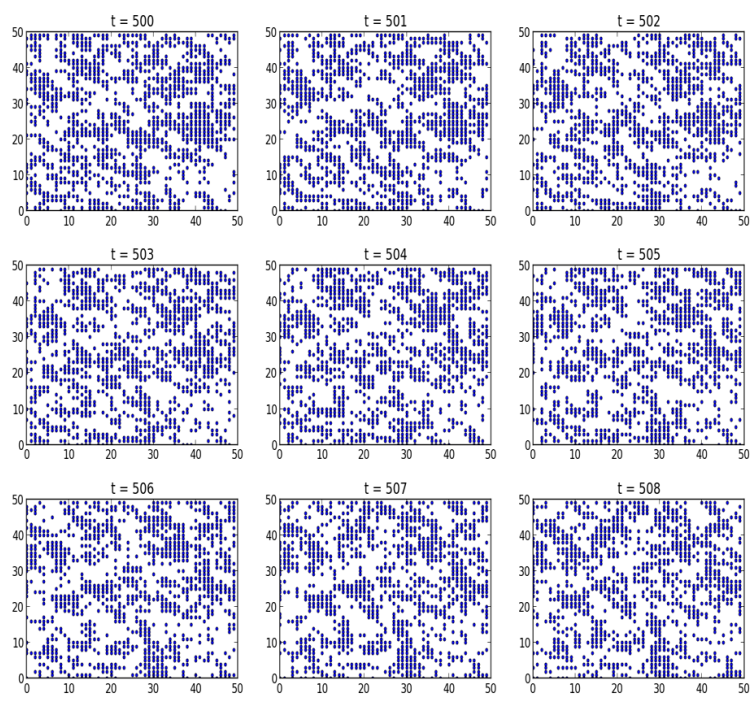

(a) $J=0.5$
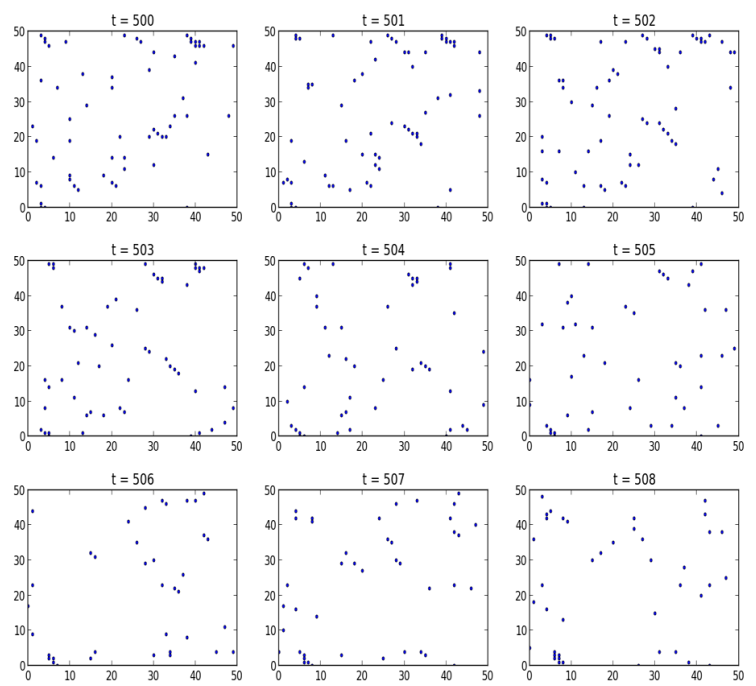

(b) $J=1.0$

Figure 3: Simulation of nine successive steps of the PCA dynamics defined by $2.5,2.6$ and 3.15 , with $N=50, q=0.5$ and different values of $J$. Blue dots represents +1 spins. Figure (b) shows a negative spontaneous magnetisation which is not observed in (a) 

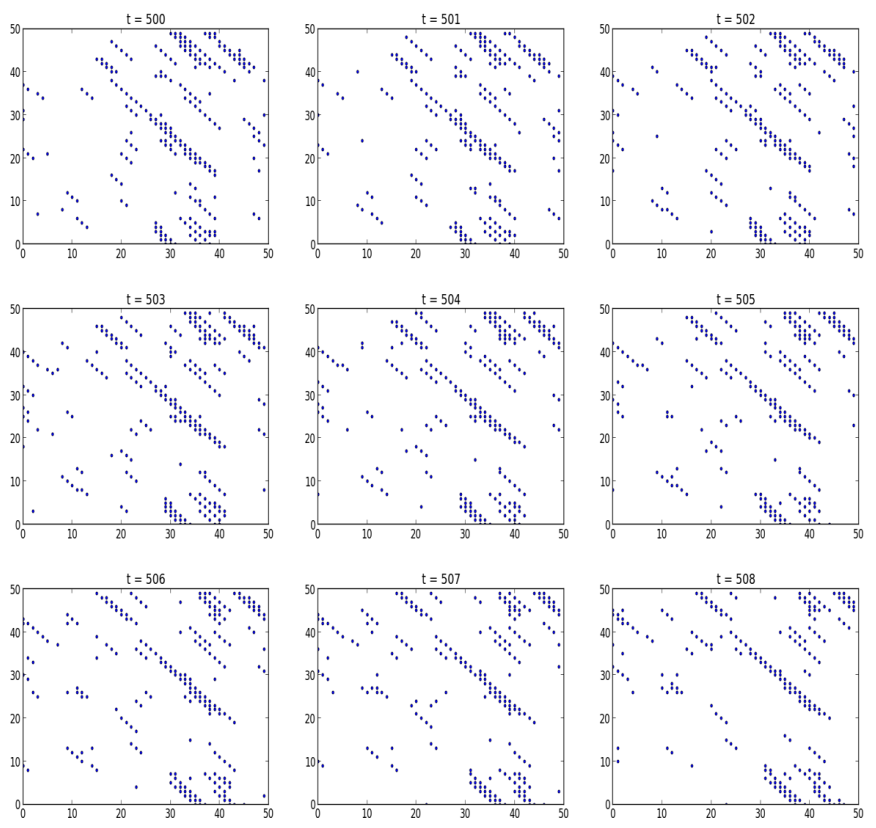

Figure 4: Simulation of nine successive steps of the PCA dynamics defined by $2.5,, 2.6$ and $(3.15)$, with $N=50, J=1.5$ and $q=0.1$. Blue dots represents +1 spins.

\section{ACKNOWLEDGMENTS}

The first author would like to express appreciation to Alexander Agathos (West University of Timisoara) and Salvatore Filippone (University of Rome "Tor Vergata") for the useful comments regarding the CUDA implementation; he would also like to address a very special thank to Thomas Falch (NTNU) and Johannes Kvam (NTNU) for their gargantuan help in developing and running the code.

REFERENCES

[Bre99] Pierre Bremaud. Markov chains: Gibbs fields, Monte Carlo simulation, and queues, volume 31. springer, 1999.

[CNo3] Emilio NM Cirillo and Francesca R Nardi. Metastability for a stochastic dynamics with a parallel heat bath updating rule. Journal of statistical physics, 110(1-2):183-217, 2003.

[DPSS12] Paolo Dai Pra, Benedetto Scoppola, and Elisabetta Scoppola. Sampling from a gibbs measure with pair interaction by means of pca. Journal of Statistical Physics, 149(4):722-737, 2012.

[GC95a] G Gallavotti and EGD Cohen. Dynamical ensembles in nonequilibrium statistical mechanics. Physical Review Letters, 74(14):2694, 1995.

[GC95b] Giovanni Gallavotti and EGD Cohen. Dynamical ensembles in stationary states. Journal of Statistical Physics, 8o(5-6):931-970, 1995.

[GKLM89] Sheldon Goldstein, Roelof Kuik, Joel L Lebowitz, and Christian Maes. From pca's to equilibrium systems and back. Communications in mathematical physics, 125(1):71-79, 1989.

[Hägo2] Olle Häggström. Finite Markov chains and algorithmic applications, volume 52. Cambridge University Press, 2002. 
[ISSo7] Antonio Iovanella, Benedetto Scoppola, and Elisabetta Scoppola. Some spin glass ideas applied to the clique problem. Journal of Statistical Physics, 126(4-5):895-915, 2007.

[JLio] Giovanni Jona-Lasinio. From fluctuations in hydrodynamics to nonequilibrium thermodynamics. Progress of Theoretical Physics Supplement, 184:262-275, 2010.

[KV8o] O Kozlov and N Vasilyev. Reversible markov chains with local interaction. Multicomponent random systems, page 451, 1980.

[LMS9o] Joel L Lebowitz, Christian Maes, and Eugene R Speer. Statistical mechanics of probabilistic cellular automata. Journal of Statistical Physics, 59(1-2):117-170, 1990.

[LPWo9] David Asher Levin, Yuval Peres, and Elizabeth Lee Wilmer. Markov chains and mixing times. AMS Bookstore, 2009.

[MS93] Christian Maes and Senya B Shlosman. When is an interacting particle system ergodic? Communications in mathematical physics, 151(3):447-466, 1993.

[Sco11] Benedetto Scoppola. Exact solution for a class of random walk on the hypercube. Journal of Statistical Physics, 143(3):413-419, 2011. 